\title{
WEIGHTED HARDY TYPE INEQUALITIES ON THE HEISENBERG GROUP $\mathbb{H}^{n}$
}

\author{
ABDULlah YenER
}

\begin{abstract}
In the present article, we provide a sufficient condition on a pair of nonnegative weight functions $V$ and $W$ on the Heisenberg group $\mathbb{H}^{n}$, so that we establish a general $L^{p}$ Hardy type inequality involving these weights with a remainder term. The method we use here is practical enough to get more weighted Hardy type inequalities. We also obtain new results on two-weight Hardy and Hardy-Poincaré type inequalities with remainder terms on $\mathbb{H}^{n}$. Our findings improve and include many previously known results in special cases.
\end{abstract}

Mathematics subject classification (2010): 26D10, 22E30, 43A80.

Keywords and phrases: Heisenberg group, Hardy inequality, Hardy-Poincaré inequality.

\section{REFERENCES}

[1] B. Abdellaoui, E. Colorado And I. Peral, Some improved Caffarelli-Kohn-Nirenberg inequalities, Calc. Var. Partial Differential Equations 23 (2005), no. 3, 327-345.

[2] Adimurthi, M. Ramaswamy and N. Chaudhuri, An improved Hardy Sobolev inequality and its applications, Proc. Amer. Math. Soc. 130 (2002), 489-505.

[3] Adimurthi And A. SeKar, Role of the fundamental solution in Hardy-Sobolev-type inequalities, Proc. Roy. Soc. Edinburgh Sect. A 136 (2006), 1111-1130.

[4] J. Garcia Azorero and I. Peral Alonso, Hardy inequalities and some critical elliptic and parabolic problems, J. Differential Equations 144 (1998), no. 2, 441-476.

[5] G. Barbatis, S. FilipPas And A. Tertikas, Series expansion for Lp Hardy inequalities, Indiana Univ. Math. J. 52 (2003), no. 1, 171-190.

[6] G. Barbatis, S. FilipPas AND A. Tertikas, A unified approach to improved $L^{p}$ Hardy inequalities with best constants, Trans. Amer. Math. Soc. 356 (2004), no. 6, 2169-2196.

[7] H. BREZIS AND J. L. VAzQueZ, Blow-up solutions of some nonlinear elliptic problems, Rev. Mat. Univ. Complutense Madrid 10 (1997), 443-469.

[8] O. Calin, D. Chang And P. Greiner, Geometric Analysis on the Heisenberg Group and Its Generalizations, American Mathematical Society, International Press, 2007.

[9] C. Cowan, Optimal Hardy inequalities for general elliptic operators with improvements, Commun. Pure Appl. Anal. 9 (2010), no. 1, 109-140.

[10] L. D’ Ambrosio, Critial degenerate inequalities on the Heisenberg group, Manuscripta Math. 106 (2001), 519-536.

[11] L. D'Ambrosio, Some Hardy inequalities on the Heisenberg group, Differential Equations 40 (2004), no. 4, 552-564.

[12] L. D’ Ambrosio, Hardy-type inequalities related to degenerate elliptic differential operators, Ann. Sc. Norm. Super. Pisa Cl. Sci. 5 (2005), vol. 4, iss. 3, 451-486.

[13] M. M. FAll AND F. MAHMOUd, Weighted Hardy inequality with higher dimensional singularity on the boundary, Calc. Var. Partial Differential Equations 50 (2014), no. 3-4, 779-798.

[14] S. Filippas And A. Tertikas, Optimizing Improved Hardy Inequalities, J. Funct. Anal. 192 (2002), 186-233.

[15] G. B. Folland, A fundamental solution for a subelliptic operator, Bull. Amer. Math. Soc. 79 (1973), 373-376. 
[16] G. B. Folland AND E. M. STEIn, Estimates for the $\bar{\partial}_{b}$-complex and analysis on the Heisenberg group, Comm. Pure Appl. Math. 27 (1974), 429-522.

[17] R. FRANK AND R. SEIRINGER, Non-linear ground state representations and sharp Hardy inequalities, J. Funct. Anal. 255 (2008), no. 12, 3407-3430.

[18] N. GAROFALO AND E. LANCONELLI, Frequency functions on the Heisenberg group, the uncertainty principle and unique continuation, Ann Inst Fourier (Grenoble) 40 (1990), 313-356.

[19] F. Gazzola, H. C. Grunau And E. Mitidieri, Hardy inequalities with optimal constants and remainder terms, Trans. Amer. Math. Soc. 356 (2004), 2149-2168.

[20] N. Ghoussoub AND A. Moradifam, On the best possible remaining term in the Hardy inequality, Proc. Natl. Acad. Sci. USA 105 (2008), no. 37, 13746-13751.

[21] N. Ghoussoub And A. Moradifam, Bessel potentials and optimal Hardy and Hardy-Rellich inequalities, Math. Ann. 349 (2011), 1-57.

[22] G. R. Goldstein, J. A. Goldstein And A. Rhandi, Weighted Hardy's inequality and the Kolmogorov equation perturbed by an inverse-square potential, Appl. Anal. 91 (2011), no. 11, 2057 2071.

[23] J. A. Goldstein And I. Kombe, Nonlinear partial differential equations on the Heisenberg group, Int. J. of Evol. Eq. 1 (2005), 1-22.

[24] J. A. Goldstein AND Q. S. Zhang, On a degenerate heat equation with a singular potential, J. Func. Anal. 186 (2001), 342-359.

[25] D. HaUer AND A. RhANDI, A weighted Hardy inequality and nonexistence of positive solutions, Arch. Math. (Basel) 100 (2013), no. 3, 273-287.

[26] G. H. HARDY, Note on a theorem of Hilbert, Math. Z. 6 (1920), 314-317.

[27] I. Kомве, Hardy, Rellich and Uncertainty principle inequalities on Carnot Groups, arXiv:math.FA/0611850.

[28] I. KomBe, Sharp Hardy type inequalities on the Carnot Group, arXiv:math.FA/0501522.

[29] I. Kombe, Sharp Weighted Rellich and uncertainty principle inequalities on Carnot groups, Comm. App. Anal. 14 (2010), no. 2, 251-272.

[30] P. LindQvist, On the equation $\operatorname{div}\left(|\nabla u|^{p-2} \nabla u\right)+\lambda|u|^{p-2} u=0$, Proc. Amer. Math. Soc. 109 (1990), $157-164$.

[31] P. NiU, H. Zhang AND Y. WANG, Hardy type and Rellich type inequalities on the Heisenberg group, Proc. Amer. Math. Soc. 129 (2001), 3623-3630.

[32] I. PERAL AND J. L. VAZQUeZ, On the stability or instability of the singular solutions with exponential reaction term, Arch. Rational Mech. Anal. 129 (1995), 201-224.

[33] I. SKRZYPCZAK, Hardy-type inequalities derived from p-harmonic problems, Nonlinear Anal. 93 (2013), 30-50.

[34] Y. X. XIAO, An improved Hardy type inequality on Heisenberg group, J. Ineq. and Appl. 38 (2011).

[35] H. Zhang AND P. NiU, Hardy-type inequalities and Pohozaev-type identities for a class of pdegenerate subelliptic operators and applications, Nonlinear Anal. 54 (2003), no. 1, 165-186. 